\title{
Creencias Epistemológicas de Estudiantes de Pedagogía en Educación Parvularia, Básica y Media: ¿Diferencias en la Formación Inicial Docente?
}

\author{
Epistemological Beliefs of Preschool, Middle, and \\ High School Pre-Service Teacher Education Students: \\ Differences in Teacher Education Programs?
}

\author{
María Rosa García y Christian Sebastián \\ Pontificia Universidad Católica de Chile
}

\begin{abstract}
En el estudio que se reporta se caracterizan y comparan las creencias epistemológicas de los estudiantes de pedagogía en educación parvularia, básica y media de una universidad tradicional chilena. Los datos fueron recolectados mediante un cuestionario completado por 330 estudiantes de pedagogía, tanto de educación media, como de primer y último año de educación parvularia y básica; hombres y mujeres de edades entre 17 y 38 años. Se realizaron comparaciones de acuerdo al programa de estudio y al momento de la formación en que se encuentran los estudiantes, mediante análisis unifactorial de la varianza. Se comparó según edad mediante regresión lineal. Los resultados muestran entre los estudiantes de pedagogía las mismas diferencias encontradas en un estudio previo entre docentes en ejercicio (Guerra, 2008): los estudiantes de pedagogía en educación media presentan creencias epistemológicas significativamente más sofisticadas que los de pedagogía en educación parvularia y básica. No se encontraron diferencias significativas entre los estudiantes de primero y último año ni tampoco asociadas a la edad de los mismos. Se discute la necesidad de estudios longitudinales en esta área.
\end{abstract}

Palabras clave: formación inicial docente, creencias epistemológicas, educación

\begin{abstract}
This study describes and compares the epistemological beliefs of preschool, middle, and high school pre-service teacher education students in a Chilean university. Data were collected through a questionnaire completed by 330 student teachers training in secondary education, first and final year of preschool education and primary education. They were men and women aged 17 to 38 years. Using univariate analysis of variance, comparisons were made according to the program of study and to the point at which the students were in their studies (first or final year). Association with age was evaluated by linear regression. Student teachers show the same differences found in a previous study among in-service teachers (Guerra, 2008): student teachers in secondary education have significantly more sophisticated epistemological beliefs than students of early childhood and primary education. No significant differences were found between freshmen and seniors, nor associated with the age of the students. The need for longitudinal studies in this area is discussed.
\end{abstract}

Keywords: teacher education programs, epistemological beliefs, education

En las últimas tres décadas ha habido un cambio en el interés en las investigaciones sobre docentes, desde el estudio de las habilidades pedagógicas de los profesores en el aula hacia el estudio de sus pensamientos y creencias (Chan \& Elliott, 2000; Kagan, 1992; Pajares, 1992). Hace ya veinte años Pintrich y De Groot (1990) afirmaban que las creencias probarían constituirse como el constructo educacional más valioso de la investigación sobre profesores. ¿Por qué el auge de estudiar las creencias docentes?

Numerosas investigaciones han demostrado que las creencias de los individuos afectan en forma determinante la conducta de los mismos (Chan \& Elliott, 2004; Cho, Lee \& Jonassen,

María Rosa García y Christian Sebastián, Escuela de Psicología, Pontificia Universidad Católica de Chile, Santiago, Chile.

La correspondencia relativa a este artículo debe ser dirigida a María Rosa García, Escuela de Psicología, Pontificia Universidad Católica de Chile, Vicuña Mackenna 4860, Macul, Santiago, Chile. E-mail: mrgarci1@uc.cl 
2011; Hofer, 2004; Jervis, 2006; Pieschl, Stahl \& Bromme, 2008). Más aún, diversos estudios muestran cómo las creencias constituyen los mejores indicadores de las decisiones individuales que las personas realizan a lo largo de sus vidas (Bandura, 1986; Dweck, 1999). Esto también ocurre en el contexto pedagógico, donde el sistema de creencias de los docentes afecta fuertemente sus prácticas pedagógicas, en tanto determina las acciones y decisiones implementadas en cada momento del proceso de enseñanza-aprendizaje (Chan \& Elliott, 2000; Fullan \& Stiegelbauer, 1997).

En este contexto, un tipo de creencias que se ha visto que actúa como predictor de las prácticas que realizan los docentes son las creencias epistemológicas (Chan, 2004; Chan \& Elliott, 2000; Guerra, 2008). Estas versan sobre la naturaleza del conocimiento y del aprendizaje e influirían en casi todos los aspectos de la vida cotidiana de las personas, debido a que afectan los procesos de razonamiento, de aprendizaje y la toma de decisiones (Brownlee, 2001; Chan \& Elliott, 2004; Mason, Ariasi \& Boldrin, 2011; Schommer-Aikins, 2004; Weinstock, Neuman \& Glassner, 2006).

Para el caso de profesores chilenos en ejercicio, Guerra (2008) mostró que existe una asociación significativa y directa entre el grado de sofisticación de las creencias epistemológicas y la calidad de las prácticas docentes. El mismo estudio mostró que se encuentran diferencias significativas respecto del grado de sofisticación de las creencias epistemológicas entre docentes que se desempeñan en distintos ciclos del sistema escolar (preescolar, primario y secundario). Dado que en Chile los docentes que trabajan en cada uno de estos ciclos se forman en programas universitarios distintos, las diferencias encontradas podrían estar asociadas de algún modo con las peculiaridades de estos programas. En esta línea, la investigación que se reporta tuvo como objetivo describir y comparar las creencias epistemológicas de los estudiantes de pedagogía de una universidad chilena tradicional, según el programa de estudio y el momento de la formación en que se encuentran los estudiantes (primer o último año). Así, se orientó a responder las siguientes preguntas de investigación: ¿qué características tienen las creencias epistemológicas de los estudiantes de pedagogía de educación media y de primer y último año de educación parvularia y básica? ¿Hay diferencias entre los estudiantes que conforman estos distintos grupos en cuanto al grado de sofisticación de sus creencias epistemológicas?

\section{Antecedentes}

\section{Creencias Epistemológicas}

Existe acuerdo en que las creencias epistemológicas son concepciones personales acerca de la naturaleza del conocimiento y del aprendizaje (Chan \& Elliott, 2004; Hofer, 2001, 2002; Hofer \& Pintrich, 1997; Howard, McGee, Schwartz \& Purcell, 2000; Jehng, Johnson \& Anderson, 1993; Schommer-Aikins, 2004). Apoyados en una profunda revisión de la literatura empírica en esta área, Buehl y Alexander (2006) afirman que las creencias epistemológicas reflejan las características generales del conocimiento mismo: las epistemologías personales serían estructuras de conocimiento complejas y multidimensionales que operan diferencialmente en distintos contextos y que se transforman y desarrollan, fruto de la participación de los sujetos en prácticas culturales específicas, desde creencias denominadas ingenuas hacia creencias denominadas sofisticadas (Hofer \& Pintrich, 1997; King \& Kitchener, 2004).

En este estudio las creencias epistemológicas fueron conceptualizadas desde el punto de vista de Schommer (1990, 1994), quien sostiene que el sistema de creencias epistemológicas estaría conformado por cuatro dimensiones, cuyos polos ingenuo y sofisticado son resumidos en la Tabla 1. 
Tabla 1

Sistema de Creencias Epistemológicas

\begin{tabular}{|c|c|c|}
\hline Dimensión & Polo ingenuo & Polo sofisticado \\
\hline Estructura del conocimiento & $\begin{array}{l}\text { Conocimiento está formado por } \\
\text { piezas desordenadas }\end{array}$ & $\begin{array}{l}\text { Conocimiento es un todo } \\
\text { integrado }\end{array}$ \\
\hline Estabilidad del conocimiento & $\begin{array}{l}\text { Conocimiento es inmodificable y } \\
\text { certero }\end{array}$ & Conocimiento es tentativo \\
\hline Control del aprendizaje & $\begin{array}{l}\text { Capacidad para aprender está } \\
\text { determinada desde el nacimiento }\end{array}$ & $\begin{array}{l}\text { Capacidad para aprender puede } \\
\text { incrementarse }\end{array}$ \\
\hline Velocidad del aprendizaje & $\begin{array}{l}\text { Aprendizaje ocurre rápido o no } \\
\text { ocurre }\end{array}$ & $\begin{array}{l}\text { Aprendizaje es un proceso } \\
\text { gradual }\end{array}$ \\
\hline
\end{tabular}

Otros investigadores han expandido y mejorado el modelo de Schommer (Jehng et al., 1993; Schraw, Bendixen \& Dunkle, 2002), lo cual ha permitido ampliar la evidencia que muestra una asociación directa entre operar con creencias epistemológicas sofisticadas y el logro de mejores resultados de aprendizaje en el contexto educativo. Específicamente, se han encontrado relaciones entre las creencias epistemológicas de los individuos y la comprensión de lectura y de material escrito que logran (Schommer, 1990; Schommer, Crouse \& Rhodes, 1992); el rendimiento académico que alcanzan (Jehng et al, 1993; Schommer, 1993; Schraw \& Sinatra, 2004); la interpretación de la información que efectúan (Kardash \& Howell, 2000; Quian \& Alverman, 1995); la resolución de problemas matemáticos (Muis, 2004); el logro en la solución de problemas inestructurados (Bromme \& Stahl, 2003; Cho et al., 2011; Mason et al., 2011) y el involucramiento y persistencia que muestran frente a tareas de aprendizaje (Braten \& Stromso, 2005; Buehl \& Alexander, 2006). En un nivel más general, las investigaciones muestran asociaciones entre el pensamiento de alto nivel y operar con creencias epistemológicas sofisticadas (Nussbaum \& Bendixen, 2003; Schommer-Aikins \& Hutter, 2002; Schraw, Dunkle \& Bendixen, 1995; Weinstock et al., 2006).

Estas asociaciones entre creencias epistemológicas y estrategias de aprendizaje y el pensamiento de alto nivel permitirían explicar las relaciones que se han encontrado entre las creencias epistemológicas y las prácticas docentes.

\section{Creencias Epistemológicas y Prácticas Docentes}

Docentes en ejercicio y estudiantes de pedagogía que operan con creencias epistemológicas más sofisticadas tienden a manifestar preferencia por enfoques docentes que, de modo general, pueden ser entendidos como promotores de aprendizaje en los estudiantes y/o pueden ser caracterizados como de orientación constructivista (Brownlee, 2001, 2003; Chan, 2004; Chan \& Elliot, 2000, 2002, 2004; Cheng, Chan, Tang \& Cheng, 2009; Olafson \& Schraw, 2006; Schraw \& Olafson, 2003; Sinatra \& Kardash, 2004).

Menos numerosos son los estudios que han evaluado empíricamente la asociación entre las creencias epistemológicas sostenidas por profesores en ejercicio y prácticas realizadas en la sala de clases; sin embargo, los resultados avanzan en la misma línea. En un estudio realizado con 35 profesores de ciencia, utilizando una metodología de incidente crítico, Hashweh (1996) 
mostró que aquellos docentes con creencias más sofisticadas reportaban utilizar un repertorio más variado de estrategias de enseñanza, potencialmente usar más estrategias efectivas en general y específicas para inducir cambio conceptual y valorar más dichas estrategias, comparados con profesores que sostenían creencias más ingenuas. Resultados muy similares fueron encontrados en una muestra de 21 profesores de matemáticas (Stipek, Givvin, Salmon \& MacGyvers, 2001).

En el único estudio que a la fecha ha sido llevado a cabo con una muestra numerosa, Guerra (2008) evaluó en Chile la asociación entre creencias epistemológicas y prácticas realizadas en el aula por profesores en ejercicio que postularon al Programa de Acreditación de Excelencia Pedagógica (AEP). Este programa busca identificar a aquellos docentes que en su desempeño profesional demuestran tener conocimientos, habilidades y competencias de excelencia. Los docentes acreditados reciben una retribución económica y el reconocimiento entre sus pares. Los docentes que postulan a este programa son evaluados a través de una prueba de conocimientos disciplinarios y de un portafolio, el cual incluye, entre otras evidencias presentadas por el docente, la video-grabación de una clase a su cargo. Para este estudio, los docentes respondieron además a un cuestionario de creencias epistemológicas. En una muestra de 1.634 profesores se mostró una asociación directa entre creencias epistemológicas sofisticadas y "prácticas docentes que promueven oportunidades de aprendizaje equitativas y de calidad" e "implican retroalimentación sistemática a las intervenciones de los estudiantes". Estas son buenas prácticas que el programa AEP evalúa en los videos presentados por los profesores. El estudio mostró además que, en promedio, las creencias que sostienen estos docentes acerca del conocimiento tienden a acercarse al polo ingenuo y que su grado de sofisticación depende del ciclo en que se desempeñan. Los docentes que ejercen en primer y segundo ciclo de educación básica son quienes presentan creencias más ingenuas y, en contraposición, los docentes de media son quienes presentan creencias más sofisticadas.

No habiendo evidencia previa que haya mostrado este tipo de diferencias, la autora avanza dos hipótesis explicativas. Por una parte, las condiciones institucionales del ejercicio docente son diferentes: en la educación secundaria los profesores trabajan en torno a una disciplina del saber, por lo que pueden profundizar en ella, e interactúan con estudiantes que, por su nivel de desarrollo cognitivo general, se encuentran probablemente en un nivel de mayor sofisticación que los estudiantes del ciclo primario. Por otra parte, en Chile quienes ejercerán como docentes en ciclo preescolar y primario se forman como profesores generalistas, en programas que enfatizan conocimientos disciplinares básicos, sin especializaciones en áreas disciplinares específicas. Por el contrario, los estudiantes de educación media se forman como licenciados en una disciplina determinada y, posteriormente, ingresan al programa de pedagogía media donde se forman en conocimientos pedagógicos (Cox, 2007, Octubre). Podría hipotetizarse, entonces, que la formación que profundiza en áreas disciplinares específicas promueve la sofisticación de creencias epistemológicas, lo cual no ocurriría en las formaciones de los estudiantes de ciclos preescolar y primario.

Para clarificar y sopesar estas hipótesis resulta necesario revisar algunos elementos concernientes al desarrollo de las creencias epistemológicas y su asociación con las prácticas educativas.

\section{Formación Docente y Cambios en Creencias}

¿Cómo evolucionan las creencias epistemológicas desde la ingenuidad a la sofisticación? En los estudios iniciales se subrayó la estabilidad de las creencias, enfatizando que mientras más tempranamente una creencia es incorporada al sistema más difícil es alterarla y que, además, algunas creencias tenderán a auto-perpetuarse, perseverando incluso en contradicción con el conocimiento adquirido a través de la experiencia y la educación (Chin \& Brewer, 1993; Pajares, 1992). Sin embargo, ya desde los estudios originales de Perry (1970) y los trabajos de Schommer (1990, 1993, 1994, 1998; Schommer-Aikins, 2004) se afirmaba que las creencias 
epistemológicas se transforman desde una postura más bien ingenua a una más sofisticada a medida que los estudiantes avanzaban en su formación universitaria.

Producto de la revisión de grandes líneas de investigación empírica, Hofer y Pintrich (1997; Hofer, 2001) señalan que existe evidencia para afirmar una tendencia general de desarrollo hacia la sofisticación al avanzar los sujetos en edad y, en particular, en experiencias educativas. Kuhn, Cheney y Weinstock (2000) precisan el sentido de esta secuencia de desarrollo al confirmar, en una muestra de siete grupos diferentes de niños, adolescentes y adultos que variaban en educación, edad y experiencia vital, que la comprensión epistemológica avanza en todos los casos desde una posición en que predomina la dimensión objetiva del conocimiento a una en que prima la dimensión subjetiva, para luego avanzar a un tercer momento en que se busca permanentemente una coordinación entre ambas dimensiones.

Estudios más recientes destacan que el cambio de creencias epistemológicas se produce en la articulación de mecanismos psicológicos del sujeto y de su interacción social en contexto (Baxter Magolda, 2004; Pintrich, 2002). Esto lleva a subrayar que el desarrollo hacia la sofisticación de creencias se produce solo bajo ciertas condiciones como, por ejemplo, a través de la participación en experiencias educativas de alto nivel —en particular, la formación de postgrado- (Jehng et al., 1993). De modo más específico, Tillema (1998) y Bendixen y Rule (2004) proponen que el cambio en las creencias se produce gracias a una elaboración profunda de las preconcepciones que traen los estudiantes a la formación. Los dispositivos de formación que confrontan sistemáticamente estas concepciones previas, para luego presentar conceptos nuevos que permiten una mejor comprensión de la realidad, son aquellos que lograrían vencer la tendencia de los estudiantes a resguardar sus preconcepciones a través de distintos mecanismos (Buehl \& Alexander, 2006; Jehng et al., 1993; Kuhn et al., 2000; Tillema, 1998). Los estudios revisados por Muis (2004) en el área de la formación inicial o permanente de profesores en matemáticas muestran esta misma tendencia. Asimismo, Gill, Ashton y Algina (2004), en un estudio experimental con 161 estudiantes de pedagogía en matemáticas, encontraron una sofisticación de las creencias epistemológicas solo en la condición en que estas fueron específicamente activadas y desafiadas. Brownlee (2003; Brownlee, Purdie \& Boulton-Lewis, 2001) muestra resultados similares en el área de la formación de educadores preescolares. Por desgracia, la formación habitual de los docentes tiende a no afectar las creencias con que los estudiantes de pedagogía inician dicha formación (Murphy, Delli \& Edwards, 2004).

En Chile son escasas las experiencias de dispositivos orientados intencionalmente al desarrollo de creencias sofisticadas entre los profesores (Gómez, Santa Cruz, Thomsen \& Rodríguez, 2008). Más aún, el Informe de la Comisión sobre Formación Inicial Docente elaborado el 2005 afirma que los formadores de docentes y los programas de formación inicial ignoran las creencias que los estudiantes traen consigo y, lo que es aún más relevante, hay evidencia de que estas permanecen sin mayores modificaciones, aun cuando se sabe que orientarán el desempeño del nuevo docente en el aula (Chile, Ministerio de Educación [MINEDUC], 2005). A diferencia de otras disciplinas, estos estudiantes sostienen creencias bastante sólidas acerca del conocimiento, el aprendizaje y la enseñanza, producto de su larga participación en el sistema educativo como estudiantes, junto a la fuerza que tienen los estereotipos culturales de lo que es ser un buen o mal profesor (Lumpe, Haney \& Czerniak, 2000; Sugrue, 1997).

En este contexto es posible comprender la segunda hipótesis avanzada por Guerra (2008), quien señala que la formación universitaria de los profesores preescolares y primarios, al no promover intencionalmente un cuestionamiento sistemático de las preconcepciones sostenidas acerca de la disciplina al comenzar la formación, difícilmente produciría un efecto en la sofisticación de las creencias epistemológicas. Por el contrario, el cuestionamiento sistemático acerca de las preconcepciones que los estudiantes tienen acerca de las matemáticas, la historia, la literatura o cualquiera disciplina en la cual se están formando como licenciados los futuros docentes del ciclo secundario, promovería el desarrollo de creencias epistemológicas sofisticadas.

Este estudio buscó aportar evidencia que permitiera profundizar en los resultados hallados por Guerra (2008), según el siguiente razonamiento: si la diferencia en cuanto al grado de 
sofisticación de las creencias epistemológicas de los docentes que ejercen en distintos ciclos se explica por las diferencias institucionales en el ejercicio de la profesión docente, no debería encontrarse diferencias entre los estudiantes de educación que aún no han ejercido en dichos contextos. En cambio, si esta diferencia está asociada con las distintas experiencias de formación, debiera encontrarse en estudiantes universitarios que siguen estos diferentes programas un grado mayor de sofisticación de las creencias epistemológicas de los futuros profesores de secundaria, comparados con los de primaria y preescolar.

Adicionalmente, se buscó entregar información descriptiva acerca del grado de sofisticación de las creencias epistemológicas de estudiantes de pedagogía de una universidad tradicional chilena, como una manera de superar la falta de información descrita por el Informe de la Comisión sobre Formación Inicial Docente (MINEDUC, 2005) antes mencionado. En particular, se exploró la asociación entre el grado de sofisticación de las creencias epistemológicas y: (a) el momento de la formación en que se encuentran (primero o último año) y (b) la edad de los estudiantes.

\section{Método}

\section{Participantes}

De una población de 597 estudiantes de pedagogía de primer y último año en educación parvularia y básica (ciclos preescolar y primario, respectivamente), y de primer año de educación media (ciclo secundario) de una universidad tradicional chilena, participaron 330 sujetos. En la Tabla 2 se detalla la cantidad de participantes en cada programa y año, junto con la referencia comparativa de la población. Se seleccionó una universidad que contara con programas de educación parvularia, básica y media.

La medición se realizó a todos los estudiantes durante el primer semestre del año 2008, considerándose estudiantes de primer año a quienes ingresaron a la carrera en dicho año, y de último, a quienes ingresaron el año 2005 o antes. En el caso de los estudiantes de educación media, dado que su programa se imparte con duración de un año a alumnos que han finalizado una licenciatura, todos corresponden a alumnos de ingreso 2008. No obstante, dado que ya han cursado cuatro años de formación disciplinar y este quinto año completa su formación como profesores de ciclo secundario, en las comparaciones según momento de la formación son considerados como estudiantes de último año.

Tabla 2

Participantes Según Programa, Momento de la Formación y Porcentaje de la Población

\begin{tabular}{lcccc}
\hline \multicolumn{1}{c}{ Programa } & $\begin{array}{c}\text { Momento de } \\
\text { la formación }\end{array}$ & $n$ participantes & $\begin{array}{c}N \\
\text { población }\end{array}$ & $\begin{array}{c}\text { Porcentaje de } \\
\text { la población }\end{array}$ \\
\hline Educación Parvularia & Primer año & 63 & 75 & 84,0 \\
& Último año & 33 & 112 & 30,0 \\
Educación Básica & Primer año & 117 & 14 & 82,4 \\
Educación Media & Último año & 67 & 202 & 33,1 \\
Total & & 50 & 66 & 75,8 \\
\hline
\end{tabular}


Los criterios de inclusión para los sujetos fueron estar inscrito en alguno de los tres programas de pedagogía definidos y haber ingresado al programa en el año 2005 o antes, o en el año 2008, excluyendo a los estudiantes que ingresaron en los años 2006 o 2007 y/o que se encontraran inscritos en una carrera distinta a pedagogía.

Para acceder a los participantes se contactó a las jefas de los programas de pedagogía de la universidad seleccionada, quienes facilitaron el detalle de los cursos impartidos en primer, cuarto y quinto año, junto con la nómina de alumnos inscritos en cada uno. En base a estos registros, se seleccionaron seis cursos de primer año que abarcaban toda la población de alumnos inscritos. En el caso de los alumnos de último año de párvulos y básica, dada la gran cantidad de cursos optativos, se seleccionó un número mayor de cursos. Partiendo por los cursos más numerosos, se fueron eligiendo aquellos cursos que progresivamente tenían inscritos a una mayor proporción de los estudiantes de la generación que no estaban inscritos en los cursos ya seleccionados.

Los participantes tenían edades entre los 17 y 38 años, observándose en los alumnos de primer año una frecuencia mayor de alumnos de edad entre los 18 y 19 años, y en los alumnos de último año (incluidos los de pedagogía media), una frecuencia mayor entre los 22 y 23 años.

La universidad contactada se encuentra en la ciudad de Santiago de Chile, pertenece al Consejo de Rectores y, en comparación con otras universidades de esta agrupación, posee un promedio alto de puntaje de ingreso. La población universitaria incluye estudiantes con todos los perfiles de ingreso familiar (desde un ingreso bruto familiar inferior a $\$ 278.000$ a ingresos superiores a \$2.500.000), aun cuando la mayor proporción de estudiantes corresponde a los perfiles de más altos ingresos (Valdivieso, Antivilo \& Barrios, 2006).

\section{Instrumento}

Para medir las creencias epistemológicas se utilizó un cuestionario elaborado por el área de Medición y Estudios del Programa AEP. Este instrumento se basa en el Epistemic Belief Inventory (EBI), desarrollado por Schraw et al. (2002), el cual se elaboró con el fin de mejorar la confiabilidad del Schommer Epistemological Questionnaire (SEQ). El cuestionario contempla cuatro escalas que miden las cuatro dimensiones de las creencias epistemológicas: estructura y estabilidad del conocimiento y control y velocidad del aprendizaje.

El cuestionario incluye 24 afirmaciones respecto de la naturaleza del conocimiento y del aprendizaje, frente a las cuales los individuos deben responder encerrando el número que más se acerca a su grado de acuerdo con la afirmación, usando una escala Likert de 5 puntos ( 1 significa totalmente en desacuerdo y 5, totalmente de acuerdo), en la que 1 se refiere a una creencia que se encuentra en el polo más sofisticado y 5 , una creencia que se encuentra cercana al polo ingenuo. Este cuestionario ha sido utilizado en otras investigaciones desarrolladas en el área, específicamente, en la efectuada por Guerra (2008) con profesores en ejercicio y en la de Gómez et al. (2008) con estudiantes de pedagogía.

En estudios previos este cuestionario fue sometido a análisis factoriales que apoyaron la validez de constructo de estas escalas. Este procedimiento identificó la estructura de las cuatro dimensiones propuesta originalmente como el modelo que mejor daba cuenta de los datos observados, junto con hallar correlaciones directas y estadísticamente significativas entre las escalas. Aun cuando estas magnitudes fueron moderadas, los resultados indican que cada escala logra efectivamente medir aspectos diferentes del constructo (Araya, 2005).

En el presente estudio se encontraron correlaciones directas y estadísticamente significativas entre todas las escalas. Asimismo, un análisis factorial apoyó la validez de constructo del instrumento, identificando a las cuatro escalas como el modelo que mejor se ajusta a los datos, mostrando que un 33,7\% de la varianza en el sistema de creencias epistemológicas es explicado por estas cuatro dimensiones. La Tabla 3 muestra la matriz de factores rotados. 
Tabla 3

Matriz de Factores Rotados

\begin{tabular}{|c|c|c|c|c|}
\hline $\begin{array}{c}\text { Escala } \\
\text { (pregunta) }\end{array}$ & Factor 1 & Factor 2 & Factor 3 & Factor 4 \\
\hline III_Preg17 & 0,799 & 0,117 & & \\
\hline III_Preg15 & 0,770 & & 0,165 & \\
\hline III_Preg18 & 0,759 & 0,142 & & 0,103 \\
\hline III_Preg16 & 0,521 & 0,164 & 0,146 & \\
\hline III_Preg13 & 0,474 & 0,141 & 0,108 & \\
\hline III_Preg14 & 0,472 & & & \\
\hline IV_Preg20 & 0,109 & 0,763 & & \\
\hline IV_Preg19 & & 0,631 & & \\
\hline IV_Preg23 & 0,163 & 0,535 & & 0,101 \\
\hline IV_Preg21 & 0,138 & 0,477 & 0,118 & 0,182 \\
\hline IV_Preg24 & 0,263 & 0,471 & & 0,124 \\
\hline IV_Preg22 & 0,180 & 0,352 & 0,157 & 0,187 \\
\hline II_Preg07 & & 0,129 & 0,591 & \\
\hline II_Preg10 & & & 0,569 & 0,101 \\
\hline II_Preg09 & & & 0,565 & 0,120 \\
\hline II_Preg08 & & & 0,548 & 0,130 \\
\hline II_Preg11 & & & 0,518 & 0,120 \\
\hline II_Preg12 & & & 0,315 & \\
\hline I_Preg06 & & & 0,133 & 0,561 \\
\hline I_Preg04 & & & & 0,531 \\
\hline I_Preg05 & & & & 0,503 \\
\hline I_Preg03 & & 0,181 & 0,133 & 0,495 \\
\hline I_Preg02 & & & 0,170 & 0,367 \\
\hline I_Preg01 & & & 0,114 & 0,251 \\
\hline
\end{tabular}

Nota: Valores ordenados y mayores a 0,1 .

El análisis de consistencia interna de cada escala entrega índices de alfa de Cronbach moderados (ver Tabla 4), pero por sobre los valores que han obtenido diversos cuestionarios desarrollados para medir estas creencias, que registran alfas en el rango de 0,54 a 0,76 (Schommer-Aikins, 2004).

Tabla 4

Consistencia Interna del Cuestionario de Creencias Epistemológicas

\begin{tabular}{lc}
\hline \multicolumn{1}{c}{ Escalas } & Alfa de Cronbach \\
\hline Estructura del conocimiento & 0,63 \\
Estabilidad del conocimiento & 0,70 \\
Control del aprendizaje & 0,81 \\
Velocidad del aprendizaje & 0,72 \\
\hline
\end{tabular}




\section{Procedimiento}

Se solicitó autorización para hacer el estudio a la Facultad de Educación de la institución seleccionada, contactando luego a las jefas de cada programa.

Se contactó a los profesores de los cursos seleccionados solicitándoles autorización para aplicar el cuestionario a los alumnos en su clase y se asistió a los cursos de quienes accedieron (solo uno de ellos no accedió). Allí se explicó a los estudiantes el objetivo, las características y condiciones del estudio y se les entregó una carta de consentimiento informado que, además de lo anterior, detallaba las responsabilidades de la participación y su carácter voluntario, la confidencialidad de la información entregada, la ausencia de riesgos por participar o no y los beneficios del estudio. Quienes aceptaron participar firmaron este documento. Aproximadamente un $97 \%$ de ellos accedió a participar. Este estudio no contó con la certificación de un comité de ética.

Los alumnos que aceptaron participar completaron el cuestionario de creencias epistemológicas. Durante esa aplicación los estudiantes debieron completar, además, un cuestionario de creencias acerca de la buena enseñanza, para el desarrollo de un estudio que no es reportado acá.

\section{Análisis de Datos}

Se calcularon los estadísticos descriptivos de las cuatro escalas de las creencias epistemológicas, para los tres programas de pedagogía y para los dos momentos de la formación, en los casos de educación parvularia y básica. Se realizaron comparaciones de las creencias epistemológicas de los estudiantes de pedagogía de acuerdo al programa que estudian y al momento de la formación en que se encuentran, mediante análisis unifactorial de la varianza. En este análisis se evaluó la homogeneidad de las varianzas mediante la prueba de Levene. Este supuesto se cumplió en ciertos análisis y en otros no. Cuando sí se cumplía, se utilizó el estadístico $F$ y la prueba post hoc de Tuckey y cuando no, se analizó el estadístico $F$ de Welch y la prueba post hoc de Dunnet T3.

Se efectuaron comparaciones en relación a la edad, mediante análisis de regresión lineal. En este último análisis se uso el método Enter para introducir la variable predictora (edad). Se analizó el cumplimiento de los supuestos de independencia entre los residuos, no colinealidad, normalidad y homocedasticidad de las varianzas, analizando: (a) estadístico Durbin-Watson, (b) tolerancia y factor de inflación de la varianza, (c) histograma de residuos y (d) gráfico de dispersión de residuos, respectivamente.

\section{Resultados}

\section{Análisis Sobre el Cumplimiento de Supuestos Estadísticos}

En los análisis de varianza se examinó el cumplimiento del supuesto de homogeneidad de las varianzas, frente a lo cual se procedía utilizando distintas pruebas según se cumpliera o no el supuesto, de acuerdo a lo mencionado en el Análisis de Datos.

En el análisis de regresión lineal, el análisis del cumplimiento de supuestos mostró (a) Durbin-Watson $=1,81$, indicando que no existe una seria auto-correlación entre los residuos; (b) Tolerancia $=1$ y Factor de inflación de varianza = 1, indicando ausencia de colinealidad; (c) histograma de residuos normalmente distribuido y (d) dispersión de residuos no asociados a los valores pronosticados. 


\section{Análisis Descriptivo de las Creencias Epistemológicas}

Al revisar los estadísticos descriptivos de las cuatro escalas de las creencias epistemológicas, se observó que en todos los programas y momentos de la formación las escalas referidas a la estructura y estabilidad del conocimiento presentaban una tendencia más cercana a una creencia ingenua. Por el contrario, las referidas al control y velocidad del aprendizaje presentaron tendencias más cercanas a una creencia sofisticada, tal como se muestra en la Tabla 5. Ello indicaría que los estudiantes tenderían a apreciar el conocimiento como elementos aislados unos de otros, permaneciendo estables en el tiempo, mientras que creerían que la capacidad para aprender puede incrementarse a lo largo de la vida y que el aprendizaje es un proceso gradual.

Tabla 5

Estadisticos Descriptivos de Creencias Epistemológicas

\begin{tabular}{|c|c|c|c|c|c|c|c|}
\hline Programa & $\begin{array}{l}\text { Momento de } \\
\text { la formación }\end{array}$ & Dimensión & $\mathrm{N}$ & Mínimo & Máximo & Media & $\begin{array}{l}\text { Desv. } \\
\text { típica }\end{array}$ \\
\hline \multirow{4}{*}{$\begin{array}{l}\text { Educación } \\
\text { Parvularia }\end{array}$} & \multirow{4}{*}{ Primer año } & Estructura del conocimiento & 63 & 1,30 & 4,30 & 3,06 & 0,51 \\
\hline & & Estabilidad del conocimiento & 63 & 1,33 & 4,17 & 2,57 & 0,73 \\
\hline & & Control del aprendizaje & 63 & 1,00 & 4,50 & 1,76 & 0,73 \\
\hline & & Velocidad del aprendizaje & 63 & 1,00 & 2,67 & 1,33 & 0,39 \\
\hline \multirow{4}{*}{$\begin{array}{l}\text { Educación } \\
\text { Parvularia }\end{array}$} & \multirow{4}{*}{ Último año } & Estructura del conocimiento & 33 & 1,67 & 3,70 & 2,91 & 0,48 \\
\hline & & Estabilidad del conocimiento & 33 & 1,00 & 4,00 & 2,60 & 0,58 \\
\hline & & Control del aprendizaje & 33 & 1,00 & 5,00 & 1,84 & 0,83 \\
\hline & & Velocidad del aprendizaje & 33 & 1,00 & 4,67 & 1,40 & 0,69 \\
\hline \multirow{4}{*}{$\begin{array}{l}\text { Educación } \\
\text { Básica }\end{array}$} & \multirow{4}{*}{ Primer año } & Estructura del conocimiento & 117 & 1,67 & 5,00 & 3,07 & 0,62 \\
\hline & & Estabilidad del conocimiento & 117 & 1,17 & 4,67 & 2,78 & 0,74 \\
\hline & & Control del aprendizaje & 117 & 1,00 & 3,50 & 1,84 & 0,63 \\
\hline & & Velocidad del aprendizaje & 117 & 1,00 & 3,00 & 1,36 & 0,40 \\
\hline \multirow{4}{*}{$\begin{array}{l}\text { Educación } \\
\text { Básica }\end{array}$} & \multirow{4}{*}{ Último año } & Estructura del conocimiento & 67 & 1,67 & 4,67 & 2,97 & 0,65 \\
\hline & & Estabilidad del conocimiento & 67 & 1,00 & 4,00 & 2,55 & 0,68 \\
\hline & & Control del aprendizaje & 67 & 1,00 & 3,50 & 1,69 & 0,62 \\
\hline & & Velocidad del aprendizaje & 67 & 1,00 & 42,83 & 1,26 & 0,43 \\
\hline \multirow{4}{*}{$\begin{array}{l}\text { Educación } \\
\text { Media }\end{array}$} & & Estructura del conocimiento & 50 & 1,00 & 4,17 & 2,46 & 0,71 \\
\hline & & Estabilidad del conocimiento & 50 & 1,17 & 4,17 & 2,22 & 0,73 \\
\hline & & Control del aprendizaje & 50 & 1,00 & 3,67 & 1,66 & 0,63 \\
\hline & & Velocidad del aprendizaje & 50 & 1,00 & 2,00 & 1,19 & 0,27 \\
\hline
\end{tabular}

\section{Análisis Comparativo de las Creencias Epistemológicas}

En relación al programa: pedagogía en educación parvularia, básica o media. Al comparar las creencias epistemológicas de los estudiantes de acuerdo al programa que cursan, se observa que existen diferencias significativas en las escalas estructura del conocimiento, $F$ de Welch $(2,123,9)=14,20, p<0,001$ (Levene $=4,97, p=0,007$ ), estabilidad del conocimiento, $F(2,327)=9,10, p<0,001$ (Levene $=0,31, p=0,73)$, y velocidad del aprendizaje, $\mathrm{F}$ de Welch $(2,149,8)=5,13, p=0,007$ (Levene $=3,26, p=0,04)$. Las comparaciones post hoc mostraron que en las escalas estructura del conocimiento, estabilidad del conocimiento y velocidad del aprendizaje los estudiantes de educación parvularia y básica presentan niveles significativamente más ingenuos que los estudiantes de educación media. 
Respecto de la magnitud de las diferencias, se encontró que en la escala estructura del conocimiento el tamaño del efecto es de $\eta^{2}=0,102,95 \%$ ICs $[2,91,3,13]$ y $[2,26,2,66]$ para los estudiantes de educación parvularia y básica y los estudiantes de educación media, respectivamente; en la escala estabilidad del conocimiento el tamaño del efecto es de $\eta^{2}=0,053,95 \%$ ICs $[2,44,2,80]$ y $[2,00,2,42]$ para los primeros y segundos, respectivamente; y en la escala velocidad del aprendizaje el tamaño del efecto es de $\eta^{2}=0,016,99 \%$ ICs $[1,25,1,46]$ y $[1,11,1,26]$ para los primeros y segundos, respectivamente. En todos los casos el tamaño del efecto, según Cohen (1977), es pequeño. No obstante, las potencias asociadas dan cuenta de que las pruebas resultan capaces de detectar diferencias significativas.

$\mathrm{Al}$ replicar estos análisis excluyendo de la muestra a los estudiantes de primer año de formación, los resultados se mantienen, a excepción de la significación observada en la dimensión velocidad del aprendizaje, efecto que desaparece. Estos últimos resultados dan cuenta de que, al comparar a los estudiantes de último año de párvulos y básica con los estudiantes de media, las diferencias de acuerdo al programa de estudios se mantienen, observándose creencias epistemológicas relacionadas con la estructura y estabilidad del conocimiento significativamente más sofisticadas en los estudiantes de pedagogía media $(p<0,05)$.

En relación al momento de la formación: estudiantes en primer y en último año. Al comparar las creencias epistemológicas de los estudiantes de acuerdo al momento de la formación en que se encuentran, se observan diferencias significativas en las escalas estructura del conocimiento, $F$ de Welch $(1,295,4)=16,24, p<0,001$ (Levene = 5,05, $p=0,02$ ) y estabilidad del conocimiento, $F(1,328)=10,46, p=0,001$ (Levene $=0,40, p=0,53$ ). Los resultados indican que en ambas escalas los estudiantes de primer año presentan creencias significativamente más ingenuas ( $M=3, D E=0,5$ en estructura; $M=2,7, D E=0,7$ en estabilidad) que los de último año ( $M=2,7, D E=0,6$ en estructura; $M=2,4, D E=0,7$ en estabilidad).

Sin embargo, al comparar de acuerdo al momento de la formación en que se encuentran los alumnos, excluyendo de la muestra a los de educación media, los resultados indican que no existen diferencias significativas entre los estudiantes de primer y último año en ninguna de las dimensiones de las creencias epistemológicas.

En relación a la edad de los participantes: entre 17 y 38 años. Los resultados muestran un solo modelo que resulta estadísticamente significativo, en el cual la edad explica un $1,8 \%$ de la varianza de la escala estructura del conocimiento, $R^{2}=0,018, F(1,328)=5,96$, $p=0,015, \beta=-0,13, p=0,015,95 \%$ IC $[3,05,3,98]$. El tamaño del efecto asociado es pequeño $\left(\eta^{2}=0,069\right)$.

\section{Discusión}

En general, los resultados encontrados son consistentes con los de Guerra (2008) y entregan evidencia en la línea de descartar la hipótesis de que las diferencias en cuanto al grado de sofisticación de las creencias epistemológicas entre docentes que se desempeñan en distintos ciclos del sistema educativo se explican exclusivamente por las diferencias institucionales en el ejercicio de la profesión docente. Evidentemente, no se puede descartar que las condiciones de trabajo de los profesores que se desempeñan en el ciclo secundario tengan algún efecto promotor de las creencias epistemológicas sofisticadas, comparadas con las de los docentes que trabajan en los ciclos preescolar y primario. Sin embargo, si la diferencia se encuentra ya entre los estudiantes de pedagogía, es posible suponer que al menos parte de los factores que la explican son distintos (y probablemente previos) a aquellos que dependen de las diferentes culturas organizacionales de los ciclos del sistema educativo.

La segunda hipótesis planteada por Guerra (2008), que haría depender el distinto grado de sofisticación de las creencias epistemológicas de las diferencias de los programas de formación docente, pareciera recibir cierto apoyo de los resultados del presente estudio. En efecto, una primera mirada a la comparación entre las creencias epistemológicas de los estudiantes de 
primer y último año de educación parvularia y básica parecería confirmar la predicción de que estos programas, al no cuestionar sistemáticamente las preconcepciones de los estudiantes, no ejercerían un efecto de sofisticación de sus creencias epistemológicas. Sin embargo, el diseño de este estudio no permite establecer conclusiones sobre los efectos de la formación en las creencias de los estudiantes. Para concluir en forma certera respecto de esto se requeriría un estudio de carácter longitudinal.

Otra hipótesis que puede plantearse para interpretar las diferencias observadas es que las creencias epistemológicas pueden estar influenciadas por un efecto de auto-selección: estudiantes con creencias más sofisticadas optarían por proseguir estudios de licenciaturas diversas, distintos a pedagogía, a diferencia de estudiantes con creencias más ingenuas, quienes ingresarían directamente a las pedagogías en educación básica y parvularia. Este efecto podría constituir, a su vez, un efecto que la formación escolar tendría sobre los estudiantes. Futuros estudios de diseño longitudinal podrán indagar en esta materia.

Por otra parte, este estudio no encontró diferencias significativas en las creencias epistemológicas de los participantes diferenciados según su edad, a pesar de que otros estudios han hallado diferencias en muestras de población adulta (Schommer, 1998) y en una muestra de docentes chilenos en ejercicio (Guerra, 2008). Una posible explicación apunta a que el rango de edades de los participantes de este estudio es pequeño. Probablemente ello incida en la dificultad para hallar diferencias significativas, aun cuando la edad resulte un elemento diferenciador de las creencias epistemológicas de los individuos.

En términos de la formación de profesores en Chile, este estudio reafirma la necesidad de prestar una mayor atención al desarrollo de las creencias epistemológicas de los futuros docentes. Como se mencionó en los antecedentes de esta investigación, existe evidencia que muestra que cuando los docentes sostienen creencias epistemológicas sofisticadas, sus prácticas pedagógicas en el aula son de un mayor nivel y promueven mejores y más profundos aprendizajes en sus estudiantes (Chan 2004; Chan \& Elliott, 2000; Guerra, 2008; Kang \& Wallace, 2005). El hecho de que estudiantes de pedagogía de último año sostengan creencias epistemológicas más bien ingenuas hace prever que sus prácticas en el aula no tendrán toda la fuerza transformadora que la sociedad espera, en particular, si se recuerda que, como tendencia general, los profesores en ejercicio también sostienen creencias epistemológicas que tienden al polo de la ingenuidad. Evidentemente, las prácticas docentes dependen de múltiples factores; sin embargo, los centros de formación docente pueden hacer más probables ciertas prácticas docentes futuras a través de una preocupación activa y eficaz por desarrollar creencias epistemológicas sofisticadas en sus estudiantes de pedagogía. Aun con la limitante de que este estudio presenta la realidad de una sola universidad, sus resultados hacen pensar que todavía hay mucho por hacer en este terreno. Probablemente, un esfuerzo inicial que pueden hacer estos centros es investigar empíricamente cuál es el nivel de desarrollo de las creencias epistemológicas de sus estudiantes y cómo estas varían a lo largo de la formación, con miras a poner en marcha dispositivos de enseñanza-aprendizaje que potencien a los futuros profesores en su comprensión del aprender y el enseñar.

\section{Referencias}

Araya, C. (2005). Informe para estudio de validación AEP 2005. Manuscrito no publicado, Centro de Medición MIDE UC, Santiago, Chile.

Bandura, A. (1986). Social foundations of thought and action: A social cognitive theory. Englewood Cliffs, NJ: Prentice Hall.

Baxter Magolda, M. B. (2004). Evolution of a constructivist conceptualization of epistemological reflection. Educational Psychologist, 39, 31-42. doi:10.1207/s15326985ep3901_4

Bendixen, L. D. \& Rule, D. C. (2004). An integrative approach to personal epistemology: A guiding model. Educational Psychologist, 39, 69-80. doi:10.1207/s15326985ep3901_7

Braten, I. \& Stromso, H. I. (2005). The relationship between epistemological beliefs, implicit theories of intelligence, and self-regulated learning among Norwegian postsecondary students. British Journal of Educational Psychology, 75, 539-565. doi:10.1348/000709905X25067 
Bromme, R. \& Stahl, E. (2003). The impact of epistemological beliefs on e-learning: The case of help-seeking. En F. W. Hesse \& Y. Tamura (Eds.), The joint workshop of cognition and learning through media-communication for advanced e-learning (pp. 29-35). Berlin, Alemania: Deutsch-Japanisches Zentrum.

Brownlee, J. M. (2001). Knowing and learning in teacher education: A theoretical framework of core and peripheral beliefs in application. Asia-Pacific Journal of Teacher Education \& Development, 4, 131-155.

Brownlee, J. (2003). Paradigm shifts in pre-service teacher education students: Case studies on changes in epistemological beliefs. Australian Journal of Educational \& Developmental Psychology, 3, 1-6.

Brownlee, J., Purdie, N. \& Boulton-Lewis, G. (2001). Changing epistemological beliefs in pre-service teacher education students. Teaching in Higher Education, 6, 247-268. doi:10.1080/13562510120045221

Buehl, M. M. \& Alexander, P. A. (2006). Examining the dual nature of epistemological beliefs. International Journal of Educational Research, 45, 28-42. doi:10.1016/j.ijer.2006.08.007

Chan, K. (2004). Preservice teacher's epistemological beliefs and conceptions about teaching and learning: Cultural implications for research in teacher education. Australian Journal of Teacher Education, 29(1), Artículo 1. Extraído de http://ro.ecu.edu.au/ajte/vol29/iss1/1

Chan, K.-W. \& Elliott, R. G. (2000). Exploratory study of epistemological beliefs of Hong Kong teacher education students: Resolving conceptual and empirical issues. Asia-Pacific Journal of Teacher Education, 28, $225-234$. doi:10.1080/713650691

Chan, K.-W. \& Elliot, R. (2002). Exploratory study of Hong Kong teacher education students' epistemological beliefs: Cultural perspectives and implications on beliefs research. Contemporary Educational Psychology, 27, $392-414$. doi:10.1006/ceps.2001.1102

Chan, K.-W. \& Elliott, R. (2004). Relational analysis of personal epistemology and conceptions about teaching and learning. Teaching and Teacher Education, 20, 817-831. doi:10.1016/j.tate.2004.09.002

Cheng, M. M. H., Chan, K.-W., Tang, S. Y. F. \& Cheng, A. Y. N. (2009). Pre-service teacher education students' epistemological beliefs and their conceptions of teaching. Teaching and Teacher Education, 25, 319-327. doi:10.1016/j.tate.2008.09.018

Chile, Ministerio de Educación (2005). Informe Comisión Sobre Formación Inicial Docente. Santiago, Chile: Autor.

Chin, C. A. \& Brewer, W. F. (1993). The role of anomalous data in knowledge acquisition: A theoretical framework and implications for science instruction. Review of Educational Research, 63, 1-49. doi:10.3102/00346543063001001

Cho, Y. H., Lee, J. \& Jonassen, D. H. (2011). The role of tasks and epistemological beliefs in online peer questioning. Computers \& Education, 56, 112-126. doi:10.1016/j.compedu.2010.08.014

Cohen, J. (1977). Statistical power analysis for the behavioral sciences. New York, NY: Academic Press.

Cox, C. (2007, Octubre). Saber pedagógico: desafíos urgentes en formación inicial de profesores. Ponencia presentada en el Seminario de Evaluación Docente en Chile, Centro de Medición MIDE UC, Pontificia Universidad Católica de Chile, Santiago, Chile.

Dweck, C. (1999). Self-theories: Their role in motivation, personality and development. Philadelphia, PA: Psychology Press.

Fullan, M. G. \& Stiegelbauer, S. (1997). El cambio educativo: guía de planeación para maestros. México DF, México: Trillas.

Gill, M. G., Ashton, P. T. \& Algina, J. (2004). Changing preservice teachers' epistemological beliefs about teaching and learning in mathematics: An intervention study. Contemporary Educational Psychology, 29, 164-185. doi:10.1016/ j.cedpsych.2004.01.003

Gómez, V., Santa Cruz, J., Thomsen, P. \& Rodríguez, C. (2008). Diseño, aplicación y análisis de una intervención para elevar la calidad del aprendizaje en el aula a partir del cambio conceptual del profesor sobre sus prácticas pedagógicas constructivas (Proyecto FONDECYT 2007/1070798). Santiago, Chile: Pontificia Universidad Católica de Chile, Facultad de Educación, Departamento de Psicopedagogía y Orientación.

Guerra, P. (2008). Creencias epistemológicas y de eficacia docente de profesores que postulan al Programa de Acreditación de Excelencia Pedagógica y su relación con las prácticas de aula (Tesis de Magíster no publicada), Escuela de Psicología, Pontificia Universidad Católica de Chile, Santiago, Chile.

Hashweh, M. Z. (1996). Effects of science teachers' epistemological beliefs in teaching. Journal of Research in Science Teaching, 33, 47-63. doi:10.1002/(SICI)1098-2736(199601)

Hofer, B. K. (2001). Personal epistemology research: Implications for learning and teaching. Educational Psychology Review, 13, 353-383. doi:10.1023/A:1011965830686

Hofer, B. K. (2002). Personal epistemology as a psychological and educational construct: An introduction. En B. K. Hofer \& P. R. Pintrich (Eds.), Personal epistemology: The psychology of beliefs about knowledge and knowing (pp. 3-14). Mahwah, NJ: Lawrence Erlbaum Associates.

Hofer, B. K. (2004). Exploring the dimensions of personal epistemology in differing classroom contexts: Student interpretations during the first year of college. Contemporary Educational Psychology, 29, 129-163. doi:10.1016/ j.cedpsych.2004.01.002

Hofer, B. K. \& Pintrich, P. R. (1997). The development of epistemological theories: Beliefs about knowledge and knowing and their relation to learning. Review of Educational Research, 67, 88-140. doi:10.3102/00346543067001088

Howard, B. C., McGee, S., Schwartz, N. \& Purcell, S. (2000). The experience of constructivism: Transforming teacher epistemology. Journal of Research on Computing in Education, 32, 455-465.

Jehng, J.-C. J., Johnson, S. D. \& Anderson, R. C. (1993). Schooling and students' epistemological beliefs about learning. Contemporary Educational Psychology, 18, 23-35. doi:10.1006/ceps.1993.1004 
Jervis, R. (2006). Understanding beliefs. Political Psychology, 27, 641-663. doi:10.1111/j.1467-9221.2006.00527.x

Kagan, D. M. (1992). Implication of research on teacher belief. Educational Psychologist, 27, 65-90. doi:10.1207/ s15326985ep2701_6

Kang, N.-H. \& Wallace, C. S. (2005). Secondary science teachers' use of laboratory activities: Linking epistemological beliefs, goals and practices. Science Education, 89, 140-165. doi:10.1002/sce.20013

Kardash, C. M. \& Howell, K. L. (2000). Effects of epistemological beliefs and topic specific beliefs on undergraduates' cognitive and strategic processing of dual positional text. Journal of Educational Psychology, 92, 524-535. doi:10.1037//0022-0663.92.3.524

King, P. M. \& Kitchener, K. S. (2004). Reflective judgment: Theory and research on the development of epistemic assumptions through adulthood. Educational Psychologist, 39, 5-18. doi:10.1207/s15326985ep3901_2

Kuhn, D., Cheney, R. \& Weinstock, M. (2000). The development of epistemological understanding. Cognitive Development, 15, 309-328. doi:10.1016/S0885-2014(00)00030-7

Lumpe, A. T., Haney, J. J. \& Czerniak, C. M. (2000). Assessing teachers' beliefs about their science teaching context, Journal of Research in Science Teaching, 37, 275-292. doi:10.1002/(SICI)1098-2736(200003)

Mason, L., Ariasi, N. \& Boldrin, A. (2011). Epistemic beliefs in action: Spontaneous reflections about knowledge and knowing during online information searching and their influence on learning. Learning and Instruction, 21, 137151. doi:10.1016/j.learninstruc.2010.01.001

Muis, K. R. (2004). Personal epistemology and mathematics: A critical review and synthesis of research. Review of Educational Research, 74, 317-377. doi:10.3102/00346543074003317

Murphy, P. K., Delli, L. A. M. \& Edwards, M. N. (2004). The good teacher and good teaching: Comparing beliefs of second-grade students, preservice teachers, and inservice teachers. The Journal of Experimental Education, 72, 69-92. doi:10.3200/JEXE.72.2.69-92

Nussbaum, E. M. \& Bendixen, L. D. (2003). Approaching and avoiding arguments: The role of epistemological beliefs, need for cognition, and extraverted personality traits. Contemporary Educational Psychology, 28, 573-595. doi:10.1016/S0361-476X(02)00062-0

Olafson, L. \& Schraw, G. (2006). Teachers' beliefs and practices within and across domains. International Journal of Educational Research, 45, 71-84. doi:10.1016/j.ijer.2006.08.005

Pajares, M. F. (1992). Teachers' beliefs and educational research: Cleaning up a messy construct. Review of Educational Research, 62, 307-332. doi:10.3102/00346543062003307

Perry Jr., W. G. (1970). Forms of intellectual and ethical development in the college years: A scheme. New York, NY: Holt, Rinehart \& Winston.

Pieschl, S., Stahl, E. \& Bromme, R. (2008). Epistemological beliefs and self-regulated learning with hypertext. Metacognition and Learning, 3, 17-37. doi:10.1007/s11409-007-9008-7

Pintrich, P. R. (2002). Future challenges and directions for theory and research on personal epistemology. En B. K. Hofer \& P. R. Pintrich (Eds.), Personal epistemology: The psychology of beliefs about knowledge and knowing (pp. 389-414). Mahwah, NJ: Lawrence Erlbaum Associates.

Pintrich, P. R. \& De Groot, E. V. (1990). Motivational and self regulated learning components of classroom academic performance. Journal of Educational Psychology, 82, 33-40. doi:10.1037/0022-0663.82.1.33

Qian, G. \& Alvermann, D. (1995). Role of epistemological beliefs and learned helplessness in secondary school students' learning science concepts from text. Journal of Educational Psychology, 87, 282-292. doi:10.1037//00220663.87.2.282

Schommer, M. (1990). Effects of beliefs about the nature of knowledge on comprehension. Journal of Educational Psychology, 82, 498-504. doi:10.1037/0022-0663.82.3.498

Schommer, M. (1993). Epistemological development and academic performance among secondary students. Journal of Educational Psychology, 85, 406-411. doi:10.1037//0022-0663.85.3.406

Schommer, M. (1994). Synthesizing epistemological belief research: Tentative understandings and provocative confusions. Educational Psychology Review, 6, 293-319. doi:10.1007/BF02213418

Schommer, M. (1998). The influence of age and education on epistemological beliefs. British Journal of Educational Psychology, 68, 551-562.

Schommer, M., Crouse, A. \& Rhodes, N. (1992). Epistemological beliefs and mathematical text comprehension: Believing it is simple does not make it so. Journal of Educational Psychology, 84, 435-443. doi:10.1037/00220663.84.4.435

Schommer-Aikins, M. (2004). Explaining the epistemological belief system: Introducing the embedded systemic model and coordinated research approach. Educational Psychologist, 39, 19-29. doi:10.1207/s15326985ep3901_3

Schommer-Aikins, M. \& Hutter, R. (2002). Epistemological beliefs and thinking about everyday controversial issues. The Journal of Psychology, 136, 5-20. doi:10.1080/00223980209604134

Schraw, G., Bendixen, L. \& Dunkle, M. (2002). Development and validation of the Epistemic Belief Inventory (EBI). En B. K. Hofer \& P. R. Pintrich (Eds.), Personal epistemology: The psychology of beliefs about knowledge and knowing (pp. 261-275). Mahwah, NJ: Lawrence Erlbaum Associates.

Schraw, G., Dunkle, M. E. \& Bendixen, L. D. (1995). Cognitive processes in well-defined and ill-defined problem solving. Applied Cognitive Psychology, 9, 523-538. doi:10.1002/acp.2350090605

Schraw, G. \& Olafson, L. (2003). Teachers' epistemological world views and educational practices. Journal of Cognitive Education and Psychology, 3, 178-235. doi:10.1891/194589503787383109

Schraw, G. \& Sinatra, G. M. (2004). Epistemological development and its impact on cognition in academic domains. Contemporary Educational Psychology, 29, 95-102. doi:10.1016/j.cedpsych.2004.01.005 
Sinatra, G. M. \& Kardash, C. M. (2004). Teacher candidates' epistemological beliefs, dispositions, and views on teaching as persuasion. Contemporary Educational Psychology, 29, 483-498. doi:10.1016/j.cedpsych.2004.03.001

Stipek, D. J., Givvin, K. B., Salmon, J. M. \& MacGyvers, V. L. (2001). Teachers' beliefs and practices related to mathematics instruction. Teaching and Teacher Education, 17, 213-226. doi:10.1016/S0742-051X(00)00052-4

Sugrue, C. (1997). Student teachers' lay theories and teaching identities: Their implications for professional development. European Journal of Teacher Education, 20, 213-225. doi:10.1080/0261976970200302

Tillema, H. H. (1998). Stability and change in student teachers' beliefs about teaching. Teachers and Teaching, 4, 217 228. doi:10.1080/1354060980040202

Valdivieso, P., Antivilo, A. \& Barrios, J. (2006). Caracterización educacional y sociodemográfica de los estudiantes que rinden la PSU, postulan y se matriculan en las universidades reunidas en el Consejo de Rectores. Santiago, Chile: Consejo Nacional de Educación.

Weinstock, M. P., Neuman, Y. \& Glassner, A. (2006). Identification of informal reasoning fallacies as a function of epistemological level, grade level, and cognitive ability. Journal of Educational Psychology, 98, 327-341. doi:10.1037/0022-0663.89.2.327

Fecha de recepción: Agosto de 2010.

Fecha de aceptación: Abril de 2011. 